\title{
Association between pulmonary hypertension and an atrial septal defect
}

\author{
M. C. Post
}

Published online: 5 June 2013

(C) The Author(s) 2013. This article is published with open access at Springerlink.com

In this Journal, Lange et al. describe the use of exercise echocardiography to identify pulmonary hypertension in the presence of an atrial septal defect (ASD) [1].

\section{Atrial septal defect}

An ASD is a relatively common congenital heart disease with a birth prevalence of 1.6 per 1000 live births, and a probability of survival into adulthood of $97 \%$ [2,3]. Different types of ASDs exist, and the secundum type, located at the fossa ovalis, is the most common (75\%) [4]. An ASD is characterised by a left-to-right shunt with volume overload of the right heart and pulmonary overcirculation. This might result in arrhythmias, right heart failure, pulmonary arterial hypertension, and paradoxical embolism, the last mentioned due to a right-to-left shunt. Echocardiography is the primary diagnostic technique, and cardiac catheterisation is required in cases of a suspected increase in pulmonary artery pressure (PAP). Indication for ASD closure is the presence of a significant shunt with signs of right ventricular volume overload without the presence of a severely increased pulmonary vascular resistance (PVR) ( $<5$ Woods units) [5]. Percutaneous closure has become the first choice of treatment for secundum-type ASDs. Severe complications occurred in less than $1 \%[6]$.

\section{Pulmonary hypertension}

Pulmonary hypertension $(\mathrm{PH})$ is a haemodynamic state characterised by a mean PAP at rest of at least $25 \mathrm{mmHg}$

M. C. Post $(\bowtie)$

Department of Cardiology, St. Antonius Hospital, Koekoekslaan 1, 3435 CM Nieuwegein, the Netherlands

e-mail: m.post@antoniusziekenhuis.nl assessed by right heart catheterisation, and can be found in multiple clinical conditions [7]. The two most common are lung and left-sided heart diseases. Pulmonary arterial hypertension (PAH) is a clinical condition with pre-capillary $\mathrm{PH}$, defined as $\mathrm{PH}$ with a normal wedge pressure and cardiac output [7]. Congenital heart diseases with a systemic-topulmonary shunt are associated with PAH. The histopathological changes found in this subgroup, such as endothelial dysfunction and vascular remodelling with proliferative changes, are similar to those observed in idiopathic or other associated forms of PAH. Transthoracic echocardiography is used to estimate the presence of PH based on the tricuspid regurgitation peak velocity and additional characteristics suggestive for PH. Over- and under-estimation of the systolic PAP can occur frequently [8]. The presence of $\mathrm{PH}$ is likely if the systolic PAP is estimated at $>50 \mathrm{mmHg}$ with or without additional findings [7]. Exercise echocardiography is not recommended by the current PH guideline [7]. In addition, exercise causes an increase in mean PAP of up to $3 \mathrm{mmHg}$ per $1 \mathrm{l} / \mathrm{min}$ increase in cardiac output in healthy individuals, and this is related to age and gender $[9,10]$. The definite diagnosis of PAH should always be confirmed by right heart catheterisation.

\section{Pulmonary hypertension and ASD}

Pulmonary arterial hypertension is noted in 9 to $35 \%$ of patient with a secundum type ASD, both open and closed $[11,12]$. The definition of PH differs among the different epidemiological studies. Predictors for the occurrence of PAH are age, size of the defect, non-closed status, and female sex $[12,13]$. Pulmonary vascular disease related to the systemic-to-pulmonary shunt might be prevented by early correction of the defect in most patients. The window of opportunity for correction and prevention of PAH is lesion specific (pre- or post-tricuspid) and pulmonary vascular 
changes might still be reversible following closure. In the presence of PAH, ASD closure can be favourable if the PVR or PAP is less than two-thirds of the systemic measurements at baseline or after vasodilator challenge [5]. If the shunt has been reversed and Eisenmenger syndrome has developed, closure of the ASD is contraindicated and PAHspecific therapy should be started if the functional capacity is decreased [7].

In the study by Lange et al. 59 'asymptomatic' patients, with haemodynamically relevant primum and secundum type ASD (shunt fraction 1.7), were included [1]. Twenty-four percent suffered from atrial fibrillation and three patients had a history of stroke. Based on echocardiography, the authors stated that PH was present in $45 \%$ of the patients. However, the tricuspid regurgitation peak velocity could only be measured in $83 \%$ and additional characteristics for the presence of $\mathrm{PH}$ were not mentioned. Interestingly, the pulmonary haemodynamics (PAP and PVR) were completely normal when assessed by right heart catheterisation. This emphasises the role of echocardiography as a screening tool for $\mathrm{PH}$, and that a definite diagnosis should only be made by right heart catheterisation. Furthermore, they selected 'ad random' 27 out of 48 patients with normal to moderately elevated pulmonary pressures for stress echocardiography and described a significant increase in the estimated PAP during exercise. Unfortunately, the cardiac output and baseline characteristics of this subgroup were not mentioned. As expected, the right ventricular parameters improved and the PAP, estimated by TTE, decreased after ASD closure.

\section{Conclusion}

Pulmonary hypertension can occur in the presence of an ASD, and might be suspected by echocardiography. Currently, exercise echocardiography is not recommended for diagnosing PAH. The presence of PAH should always be confirmed by right heart catheterisation. The pulmonary haemodynamics and right ventricular function can improve after shunt closure.

Funding None.
Conflict of interests None declared.

Open Access This article is distributed under the terms of the Creative Commons Attribution License which permits any use, distribution, and reproduction in any medium, provided the original author(s) and the source are credited.

\section{References}

1. Lange SA, Braun MU, Schoen SP, et al. Latent pulmonary hypertension in atrial septal defect: dynamic stress echocardiography reveals unapparent pulmonary hypertension and confirms rapid normalisation after ASD closure. Neth Heart J. 2013. doi:10.1007/s12471-0130425-8 [Epub ahead of print].

2. van der Linde D, Konings EE, Slager MA, et al. Birth prevalence of congenital heart disease worldwide: a systematic review and meta-analysis. J Am Coll Cardiol. 2011;58(21):2241-7.

3. Moons P, Bovijn L, Budts W, et al. Temporal trends in survival to adulthood among patients born with congenital heart disease from 1970 to 1992 in Belgium. Circulation. 2010;122(22):2264-72.

4. Webb G, Gatzoulis MA. Atrial septal defects in the adult: recent progress and overview. Circulation. 2006;114(15):1645-53.

5. Baumgartner H, Bonhoeffer P, De Groot NM, et al. ESC Guidelines for the management of grown-up congenital heart disease (new version 2010). Eur Heart J. 2010;31(23):2915-57.

6. Van den Branden BJ, Post MC, Plokker HW, et al. Percutaneous atrial shunt closure using the novel Occlutech Figulla device: 6-month efficacy and safety. J Interv Cardiol. 2011;24(3):264-70.

7. Galie N, Hoeper MM, Humbert M, et al. Guidelines for the diagnosis and treatment of pulmonary hypertension. Eur Respir J. 2009;34(6):1219-63.

8. Fisher MR, Forfia PR, Chamera E, et al. Accuracy of Doppler echocardiography in the hemodynamic assessment of pulmonary hypertension. Am J Respir Crit Care Med. 2009;179(7):615-21.

9. Naeije R, Vanderpool R, Dhakal BP, et al. Exercise-induced pulmonary hypertension: physiological basis and methodological concerns. Am J Respir Crit Care Med. 2013;187(6):576-83.

10. Kovacs G, Berghold A, Scheidl S, et al. Pulmonary arterial pressure during rest and exercise in healthy subjects: a systematic review. Eur Respir J. 2009;34(4):888-94.

11. Vogel M, Berger F, Kramer A, et al. Incidence of secondary pulmonary hypertension in adults with atrial septal or sinus venosus defects. Heart. 1999;82(1):30-3.

12. Engelfriet P, Meijboom F, Boersma E, et al. Repaired and open atrial septal defects type II in adulthood: an epidemiological study of a large European cohort. Int J Cardiol. 2008;126(3):379-85.

13. Yong G, Khairy P, De Guise P, et al. Pulmonary arterial hypertension in patients with transcatheter closure of secundum atrial septal defects: a longitudinal study. Circ Cardiovasc Interv. 2009;2(5): 455-62. 